Research, Society and Development, v. 9, n. 7, e448974362, 2020

(CC BY 4.0) | ISSN 2525-3409 | DOI: http://dx.doi.org/10.33448/rsd-v9i7.4362

Florêncio, MNS, Abud, AKS, Costa, BMG \& Oliveira Jr, AM (2020). Analysis of biotechnology production and collaboration in brazil. Research, Society and Development, 9(7): 1-27, e448974362.

\title{
Análise da produção e colaboração da biotecnologia no Brasil
}

Analysis of biotechnology production and collaboration in Brazil

Análisis de la producción y colaboración de biotecnología en Brasil

Recebido: 08/05/2020 | Revisado: 09/05/2020 | Aceito: 12/05/2020 | Publicado: 21/05/2020

Márcio Nannini da Silva Florêncio

ORCID: https://orcid.org/0000-0001-5557-4181

Universidade Federal de Sergipe, Brasil

E-mail: marcio_nannini@hotmail.com

Ana Karla de Souza Abud

ORCID: https://orcid.org/0000-0001-6610-6084

Universidade Federal de Sergipe, Brasil

E-mail: ana.abud@gmail.com

Benedita Marta Gomes Costa

ORCID: https://orcid.org/0000-0002-2740-0560

Universidade Estadual Vale do Acaraú, Brasil

E-mail: martagcosta@hotmail.com

Antonio Martins de Oliveira Junior

ORCID: https://orcid.org/0000-0002-8635-7048

Universidade Federal de Sergipe, Brasil

E-mail: amartins.junior@gmail.com

\section{Resumo}

Este estudo objetivou analisar a produção e colaboração tecnológica da biotecnologia no Brasil por meio dos documentos de patentes concedidos pelo Instituto Nacional de Propriedade Industrial (INPI). A análise dos dados ocorreu mediante o uso dos indicadores de medidas de atividade tecnológica e de Análise de Redes Sociais (ARS). O panorama geral da produção biotecnológica, compreendido entre 1982 e 2016, mostrou que a maioria das patentes $(90 \%)$ é de titularidade de não residentes, com destaque para as empresas privadas. O principal detentor de biotecnologias no país são os Estados Unidos com 35,4\% dos documentos concedidos. A ARS apontou uma fraca colaboração tecnológica entre os titulares 
de patentes em biotecnologia. O estudo mostrou que os setores públicos e privados vêm respondendo às demandas apresentadas pela Política de Desenvolvimento da Biotecnologia (PDB), mas ainda é necessário o fortalecimento das políticas e ações já definidas para melhoria da bioindústria brasileira. Entre as ações indispensáveis está a disseminação da cultura de propriedade intelectual em relação à proteção da biotecnologia para as instituições públicas e o setor empresarial.

Palavras-chave: Patentes; Biotecnologia; Brasil.

\section{Abstract}

This study aimed to analyze the production and technological collaboration of biotechnology in Brazil through the patent documents granted by the National Institute of Industrial Property (INPI). Data analysis took place using the indicators of measures of technological activity and Social Network Analysis (SNA). The general panorama of biotechnological production, between 1982 and 2016, showed that the majority of patents (90\%) are owned by nonresidents, with emphasis on private companies. The main holder of biotechnologies in the country is the United States with $35.4 \%$ of the documents granted. SNA pointed to a weak technological collaboration between patent holders in biotechnology. The study showed that the public and private sectors have been responding to the demands presented by the Biotechnology Development Policy, but it is still necessary to strengthen the policies and actions already defined to improve the Brazilian bio industry. Among the indispensable actions is the dissemination of the culture of intellectual property about the protection of biotechnology for public institutions and the business sector.

Keywords: Patents; Biotechnology; Brazil.

\section{Resumen}

Este estudio tuvo como objetivo analizar la producción y la colaboración tecnológica de la biotecnología en Brasil a través de los documentos de patente otorgados por el Instituto Nacional de Propiedad Industrial (INPI). El análisis de datos se realizó utilizando los indicadores de medidas de actividad tecnológica y Análisis de Redes Sociales (ARS). El panorama general de la producción biotecnológica, entre 1982 y 2016, mostró que la mayoría de las patentes (90\%) son propiedad de no residentes, con énfasis en empresas privadas, siendo el principal titular de biotecnologías en el país Estados Unidos, con 35,4\% de los documentos otorgados. ARS señaló una débil colaboración tecnológica entre los titulares de patentes en biotecnología. El estudio mostró que los sectores público y privado han estado 
respondiendo a las demandas presentadas por la Política de Desarrollo de Biotecnología (PDB), pero aún es necesario fortalecer las políticas y acciones ya definidas para mejorar la bioindustria brasileña. Entre las acciones esenciales se encuentra la difusión de la cultura de la propiedad intelectual en relación con la protección de la biotecnología para las instituciones públicas y el sector empresarial.

Palabras clave: Patentes; Biotecnología; Brasil.

\section{Introdução}

Com a globalização e a abertura da economia brasileira nos anos noventa, o mercado nacional passou a contar com competidores estrangeiros com vasta experiência em inovação tecnológica, qualidade e preços competitivos. A indústria nacional foi, então, forçada a melhorar, a repensar suas estratégias e a impulsionar esforços para inovar e desenvolver vantagens competitivas. Nesse contexto, os direitos de propriedade intelectual se apresentam como um importante instrumento, assegurando o retorno sobre os altos investimentos em Pesquisa, Desenvolvimento e Inovação (P, D\&I) traçados, principalmente, em áreas como farmácia, química e biotecnologia, onde os gastos são elevados e as pesquisas duradouras (Belda, et al., 2014).

A biotecnologia é considerada uma importante área de inovação e desenvolvimento, pois apresenta diferentes alternativas para solucionar problemas, aprimorar produtos e processos, reduzir custos e melhorar a qualidade de vida. Seus resultados podem ser observados em diversos setores, como saúde, agroindústria e meio ambiente, e envolvem várias áreas do conhecimento, a exemplo da genética, física, química, farmacologia, medicina veterinária, alimentos, dentre outras.

Apontada como um campo em que se observa uma importância crescente da interação entre os universos da ciência, da pesquisa tecnológica e da produção industrial, a biotecnologia vem sendo utilizada em um conjunto de indicadores que medem o grau de inovação de um país (Banerjee, Gupta, \& Garg, 2000, Van Beuzekom \& Arundel, 2009, Mendes, Amorim-Borher, \& Lage, 2013). Dentre os indicadores adotados, a produção tecnológica, mais especificamente o estudo das patentes, vem sendo considerada uma forma de mensurar a inovação de um país, possibilitando conhecer a produção do conhecimento em determinada área do saber (Nelson, 2009, Tijssen, 2011).

Entre os poucos estudos que se debruçam sobre a análise da atividade de patenteamento em biotecnologia no Brasil, destacam-se os trabalhos de Dias et al. (2012), de 
Costa et al. (2012), de Buttow \& Steindel (2012), de Mendes, Borher \& Lage (2013), de Costa, Florêncio \& Oliveira Jr (2018) e de Florêncio, Abud \& Oliveira Jr (2019). Comparativamente a esses estudos, esta pesquisa, além de atualizar a compreensão sobre o desenvolvimento tecnológico da área em questão, oferece duas contribuições adicionais: (1) compara, analisa e complementa alguns dados e argumentos apresentados pela literatura citada, ao estabelecer o desenvolvimento do presente estudo sob o enfoque das áreas setoriais da biotecnologia (saúde humana, agropecuária, industrial e ambiental), instituídas na Política de Desenvolvimento da Biotecnologia (PDB), e (2) amplia a perspectiva de estudo sobre o tema, introduzindo uma análise inicial da rede de cooperação tecnológica entre os principais titulares de patentes na área.

Diante do exposto, este estudo teve por objetivo analisar a dinâmica da produção e da colaboração tecnológica da biotecnologia no Brasil a fim de indicar elementos para uma tomada de decisão governamental e empresarial que favoreçam o desenvolvimento tecnológico da biotecnologia.

\section{Metodologia}

Uma pesquisa visa obter ou alcançar novo saber ou saberes como preconiza Pereira, Shitsuka, Parreira \& Shitsuka (2018). Nesta presente pesquisa faz-se uso de bibliometria para analisar os documentos de patentes a fim de observar padrões, tendências e o desenvolvimento tecnológico ao longo dos anos, bem como indicar características da produção tecnológica em biotecnologia no Brasil. Bibliometria é um estudo quantitativo da produção científica ou tecnológica de artigos publicados em periódicos, anais de congresso ou livros (Okano, 2020), bem como de documentos de patentes.

Aliado a isso, fez-se o uso da Análise de Redes Sociais (ARS), visando compreender a cooperação tecnológica entre os agentes de inovação (academia, instituições públicas e empresas privadas) envolvidos na geração e na proteção do conhecimento em biotecnologia no país.

O dados foram coletados junto a base de patentes do Instituto Nacional de Propriedade Industrial (INPI), disponível no endereço http://www.inpi.gov.br/. Esta base de dados permite recuperar todos os documentos de patentes concedidos no Brasil. A Figura 1 apresenta o fluxograma dos procedimentos usados na coleta e análise dos dados. 
Figura 1 - Síntese do percurso metodológico.

\section{Obtenção das informações}

Coleta de dados na base de patentes do INPI por meio dos códigos CIP em biotecnologia

\section{Organização e análise de resultados}

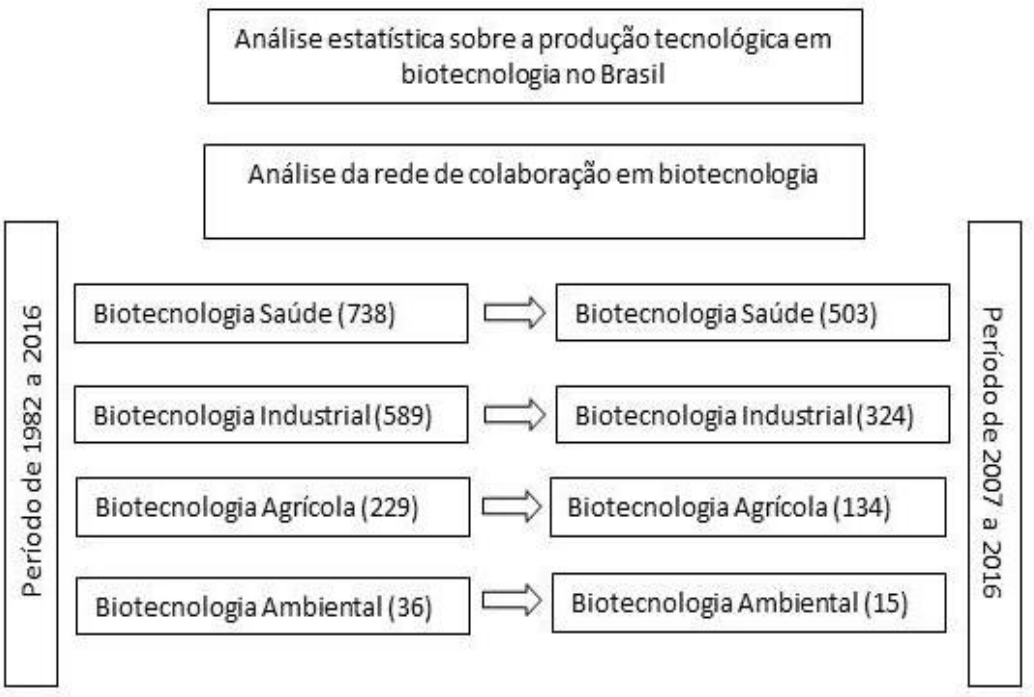

Fonte: Autores

A identificação das patentes em biotecnologia utilizou como filtro de busca os 30 códigos da Classificação Internacional de Patentes (CIP) na área apontados pela Organização para a Cooperação e Desenvolvimento Econômico (OCDE). Além destes, foi adotado o código CIP B82Y5/00, que trata das invenções em nanobiotecnologia ou nanomedicina.

A coleta dos dados ocorreu no período de março a junho de 2017, utilizando os códigos da OCDE conjuntamente com os operadores booleanos e de truncagem no campo "pesquisa avançada". Na extração dos dados foi selecionado a opção "patente concedida" e não foram adotadas restrições temporais.

Os dados extraídos foram exportados para o software Microsoft Excel® para organização, tratamento e análise das informações. O processo de análise das informações 
contou com o auxílio dos programas Ucinet versão 6.599, NetDraw versão 2.157 e Mapchart, disponível no endereço https://mapchart.net/.

\section{Resultados e Discussão}

\subsection{Panorama da Produção Tecnológica da Biotecnologia no Brasil}

O total de documentos recuperados por meio da base de dados do INPI, segundo os critérios estabelecidos na metodologia deste trabalho, foi de 1.592 patentes. Destaca-se que não foram adotadas restrições temporais, tendo-se acesso a todas as patentes concedidas pelo INPI em biotecnologia no período de 1982 a 2016.

A primeira concessão de patente na área de biotecnologia no Brasil foi realizada em 1982, referente ao pedido PI 7602164-5, intitulado "Processo para a preparação de antibiótico e composição fungicida a base deste”. O depósito da patente foi realizado pela empresa alemã Bayer AG, no ano de 1975. A partir desta data, diversos pedidos de patentes em biotecnologia foram realizados junto ao escritório do INPI, obtendo uma média de retorno de aproximadamente 45 concessões por ano.

A Figura 2 revela a evolução anual da produção tecnológica da biotecnologia no Brasil.

Figura 2 - Evolução temporal da produção tecnológica da biotecnologia no Brasil no período (a) de 1982 a 1996 e (b) de 1997 a 2016.
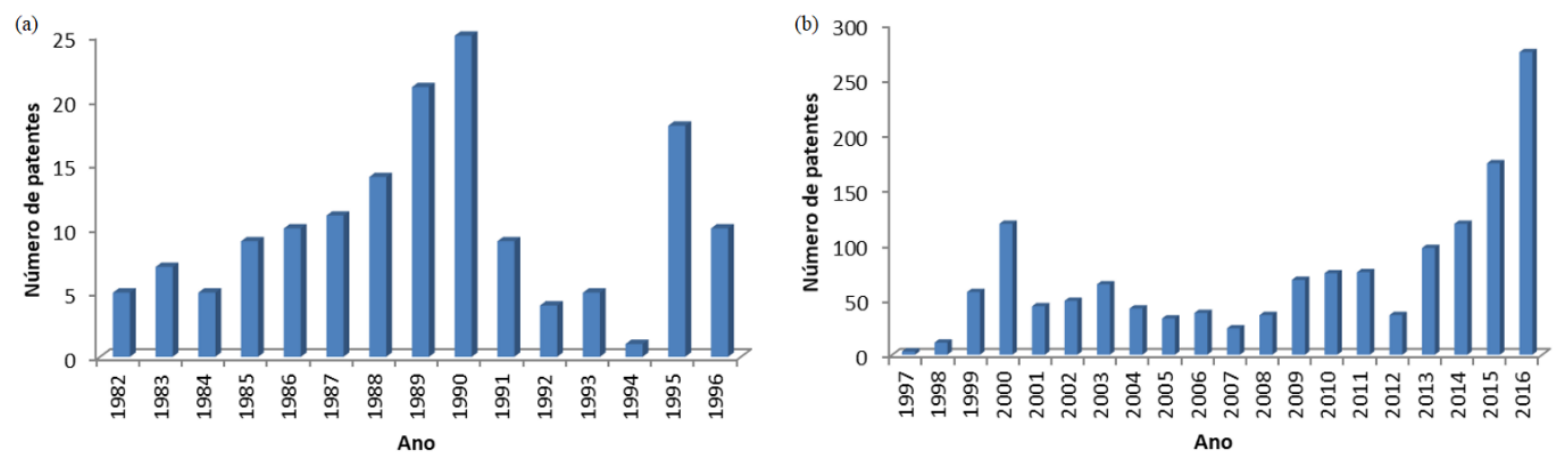

Fonte: Autores

No período anterior à entrada em vigor da Lei de Propriedade Industrial (LPI), em 1996, é possível verificar que ocorreu um pequeno número de concessões de patentes em biotecnologia (144 documentos), dos quais $78,5 \%$ foram de titularidade de não residentes e 
apenas 21,5\% foram de residentes. No período de 1982 a 1994, a taxa de crescimento em concessões de patentes se mostrou relativamente estável, com uma média de 34,3\% ao ano.

É oportuno destacar que a atual lei de patentes alterou o escopo de proteção em biotecnologia, permitindo a concessão para diversos produtos farmacêuticos e alimentícios, o que atraiu a atenção de empresas estrangeiras e expandiu os depósitos de patentes no país (Brasil, 2013). Isto justifica o recorte temporal adotado na Figura 2, que apresenta a evolução temporal das concessões de patentes antes e após a LPI.

Após implantação da LPI, observa-se um crescimento no número de concessões de patentes, com dois momentos de maior significância. O primeiro momento foi registrado nos anos de 1999 e 2000, com taxas de crescimento de 418,2\% e 108,8\%, respectivamente, em relação ao ano anterior e cerca de $63 \%$ das concessões referentes aos pedidos pipeline.

Destaca-se que as patentes pipelines tratam de um mecanismo de revalidação nacional de patentes de medicamentos, alimentos e produtos e processos químico-farmacêuticos concedidos em outros países. Estes pedidos não passam pelo exame de mérito da patenteabilidade (novidade, atividade inventiva, aplicação industrial), sendo realizado apenas uma análise formal dos documentos (Miranda, Silva, \& Pereira, 2009).

O segundo momento foi registrado no ano de 2013, com um crescimento de 169,4\%, quando foram concedidas 97 patentes contra um total de 36 documentos em 2012. Uma possível explicação para o alto crescimento observado a partir do ano de 2013 se refere à contratação de um maior número de examinadores de patentes na área de biotecnologia. Aliado a isso, foi estabelecido, no ano de 2015, uma nova diretriz de exame de pedidos de patentes em biotecnologia, com vista a aumentar a eficiência do exame no INPI (INPI, 2015).

O pequeno número de concessões ocorrido em 2012 pode estar associado ao processo de implantação do sistema de exame eletrônico de patentes iniciado em dezembro de 2010 (Matias-Pereira, 2011). Com isso, a produtividade dos examinadores reduziu consideravelmente, fato que possivelmente tenha acontecido em todas as demais áreas tecnológicas.

No período de 2000 a 2016, as patentes em biotecnologia representaram aproximadamente $2 \%$ do total das concessões no país, ficando atrás de diversos setores industriais, como química de materiais básicos, química fina, telecomunicações, entre outros (INPI, 2017). Salienta-se que o pequeno percentual de patentes concedidas na área pode estar associado, entre outros fatores, à baixa de taxa de sucesso para os pedidos de patentes (número de patentes concedidas/número de pedidos de patentes), comumente observada em 
países que apresentam um sistema de propriedade intelectual em desenvolvimento (Pinto, et al., 2017).

Embora tenham sido tomadas diversas medidas para melhorar o sistema de patente brasileiro, ainda é persistente o atraso crônico no processamento dos pedidos (Garcez Júnior \& Moreira, 2017). Nessa linha, a área de biotecnologia pode, por exemplo, apresentar um atraso ainda maior que muitos outros setores, por necessitar da prévia anuência da Agência Nacional de Vigilância Sanitária (ANVISA) para a concessão de fármacos. A Tabela 1 indica as medidas estatísticas descritivas do atraso de concessão das patentes na área biotecnologia no Brasil.

Tabela 1 - Medidas estatísticas descritivas do tempo de espera para a concessão de patentes em biotecnologia no Brasil (1982-2016).

\begin{tabular}{lccc}
\hline \multirow{2}{*}{ Estatística descritiva } & \multicolumn{3}{c}{ Grupos } \\
\cline { 2 - 4 } & Residentes & Não Residentes & Geral \\
\hline Média & 9,7 & 10,7 & 10,6 \\
Mínimo & 2,9 & 1,7 & 1,7 \\
Máximo & 18,6 & 20,3 & 20,3 \\
Desvio padrão & 3,3 & 3,7 & 3,7 \\
Coeficiente de variação & 0,340 & 0,346 & 0,349 \\
\hline
\end{tabular}

Nota: O tempo de processamento dos pedidos de patentes em biotecnologia foi calculado a partir da diferença entre a data de concessão e a data de depósito.

Fonte: Autores

O tempo médio para a concessão de patente na área de biotecnologia é de 10,6 anos, com desvio padrão de 3,7 anos. O valor mínimo encontrado referente ao período de processamento dos pedidos é de 1,7 anos, enquanto o valor máximo corresponde a 20,3 anos. Em adição, Popp, Juhl \& Johnson (2004) sugerem que a área de biotecnologia, por apresentar aplicações novas e complexas, gera uma comunicação mais frequente entre examinadores e depositantes, o que provoca um tempo de espera maior do que outros campos tecnológicos.

Os titulares residentes obtiveram um tempo de espera para concessão menor $(9,7$ anos) do que não residentes (10,7 anos). Identificou-se, também, uma alta dispersão dos dados em relação à média $(\mathrm{CV}=34,9 \%)$, sugerindo que os valores da mediana são possivelmente mais relevantes para representar o atraso das concessões na área.

Cabe frisar que o rápido processamento das patentes em biotecnologia é motivado em grande parte pelos pedidos pipeline $(12,4 \%)$, que apresentaram uma média de concessão de 5,18 anos, e pelas patentes verdes $(0,5 \%)$, que obtiveram um tempo médio de processamento 
de 4,85 anos. A maioria das patentes com período de concessão acima de 18 anos $(53,3 \%)$ correspondem à área de fármacos.

O programa "Patente Verde" foi iniciado em 2012 pelo INPI com o intuito de conceder o exame prioritário para os registros de patentes de tecnologias voltadas para o meio ambiente. Por meio do programa já foram analisados 227 pedidos de patentes até setembro de 2016, obtendo um tempo máximo de dois anos para o deferimento ou indeferimento dos depósitos (INPI, 2016).

Para Jannuzzi \& Vasconcellos (2017), este enorme atraso na concessão de patentes farmacêuticas no Brasil está associado a dois motivos principais. Primeiro, o escritório de patentes brasileiro não dispõe de servidores suficientes para a análise e tramitação dos processos administrativos.

Segundo, o fato de, por um longo período, as patentes farmacêuticas terem sido duplamente analisadas quantos aos requisitos de patenteabilidade, em virtude de não existir uma definição clara das competências técnicas do INPI e da ANVISA, o que foi superado com a assinatura da Portaria Conjunta $\mathrm{n}^{\mathrm{o}}$ 1, de 12 de abril de 2017, entre os órgãos.

A Figura 3 apresenta a distribuição do período para a concessão de patentes em biotecnologia no Brasil. Os dados mostram um longo período entre um pedido de patente em biotecnologia e sua concessão, sendo metade das patentes em biotecnologia concedidas em 12 ou mais anos e uma grande maioria (78\%) em 9 ou mais anos.

Apenas $7 \%$ das patentes foram concedidas em 43 meses ou menos, das quais $2 \% \mathrm{em}$ período inferior a 32 meses, correspondendo aos atrasos médios da concessão de patentes no European Patent Office (EPO) e United States Patent and Trademark Office (USPTO), respectivamente (Mejer \& Van Pottelsberghe De La Potterie, 2011). 
Figura 3 - Distribuição do tempo de espera para a concessão de patentes em biotecnologia no

Brasil (1982-2016).

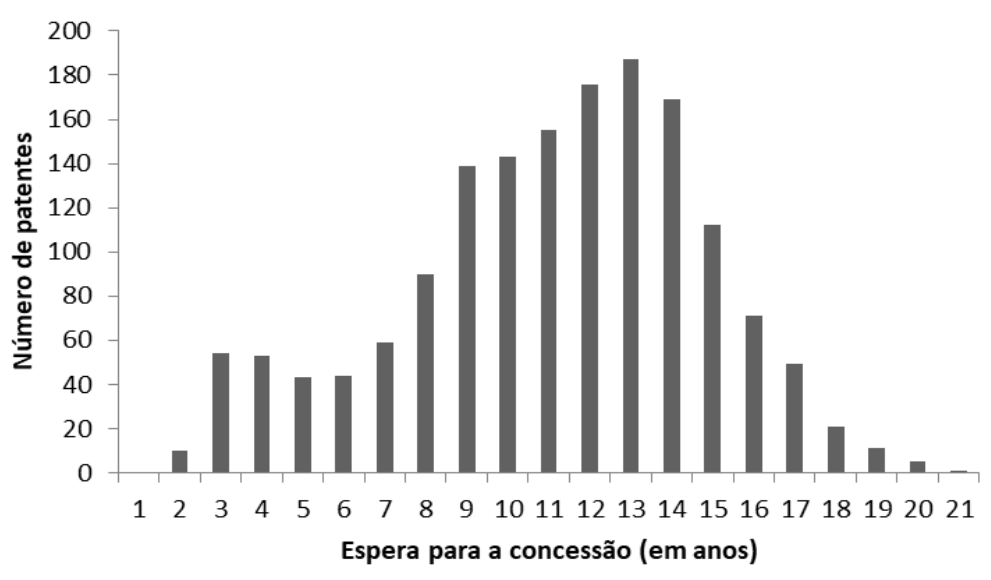

Fonte: Autores

Essa demora no processo de concessão de patentes em biotecnologia provoca efeitos desestimulantes para a bioindústria brasileira. As pequenas e médias empresas de biotecnologia ou as grandes corporações multinacionais podem diminuir seus investimentos no desenvolvimento de novas tecnologias para o país, por enfrentarem um longo período de insegurança jurídica na espera da concessão da patente. Além disso, o atraso na análise dos pedidos de patentes gera altos custos para sociedade, pois pode estender o prazo de vigência das tecnologias patenteadas.

Dos 1.592 documentos de patentes, apenas 24,5\% apresentaram dados referentes à data do pedido de exame da invenção. Essas patentes possuíram uma média de aproximadamente 38 meses entre a data de depósito e a data de início do exame. Vale mencionar que este longo período para o início do exame sofreu influência, entre outros fatores, da exigência legal do Art. 30 da LPI que determina um período de sigilo 18 (dezoito) meses contados da data de depósito ou da prioridade mais antiga (Brasil, 1996).

Cerca de 59\% das patentes em biotecnologia estão vigentes. Quanto às demais patentes, foi observado que 33,2\% foram extintas em razão da expiração do prazo de vigência, conforme disciplina o Art. 78, inciso I da LPI, 6,8\% foram extintas pela falta de pagamento da retribuição anual e 0,8\% em decorrência da renúncia do titular, caducidade (Art. 78, incisos II e III) ou pela nulidade por não ter sido atendido qualquer dos requisitos legais (Art. 50, inciso I da LPI).

Notou-se ainda que mais de $70 \%$ das patentes vigentes em biotecnologia se beneficiaram do dispositivo no parágrafo único do Art. 40 da LPI, o qual disciplina que a 
vigência de uma patente de invenção terá o prazo mínimo de 10 anos contados a partir da data de concessão.

Entre os quinze principais países de origem com mais de dez patentes concedidas em biotecnologia no Brasil, no período de 1982 a 2016 (Figura 4), nove estão localizados na Europa (Alemanha, França, Suíça, Holanda, Dinamarca, Reino Unido, Bélgica, Suécia e Itália), três na América (Estados Unidos, Brasil e Canadá), dois na Ásia (Japão e República da Coréia) e um na Oceania (Austrália).

A maioria das concessões de patentes em biotecnologia (90\%) pertence a não residentes, sendo os Estados Unidos detentor do maior número de patentes dos documentos concedidos $(35,4 \%)$, seguido pelo Brasil $(10,1 \%)$, Alemanha $(8,7 \%)$, Japão $(7,8 \%)$ e França $(6,4 \%)$. É importante destacar que o elevado número de patentes por estrangeiros é motivado pelos depósitos via Patent Cooperation Treaty (PCT), com cerca de 57\% dos registros de patentes concedidos.

Figura 4 - Mapa dos países de origem com patentes concedidas em biotecnologia no Brasil

(1982-2016).

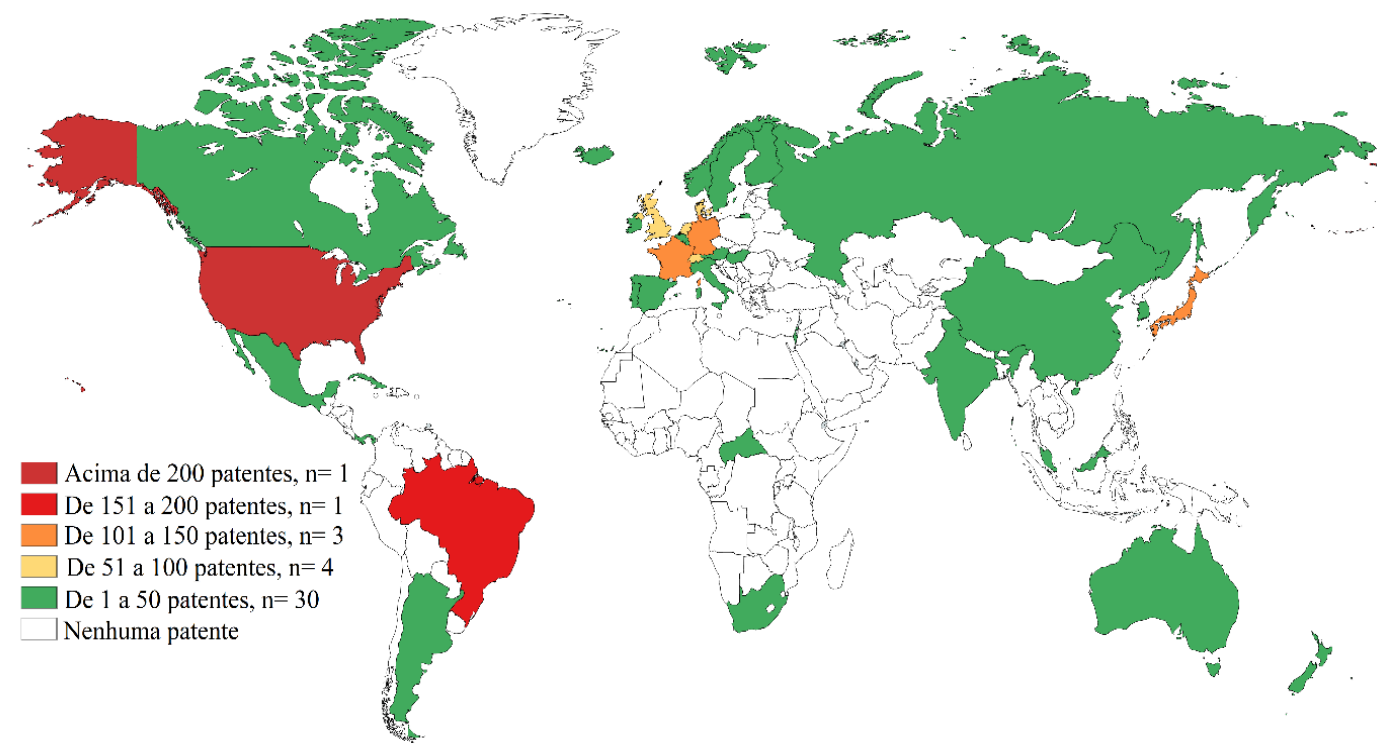

Fonte: Autores

A Figura 5 ilustra a evolução temporal das patentes concedidas em biotecnologia no Brasil entre os principais países de origem. 
Figura 5 - Evolução temporal da produção tecnológica em biotecnologia no Brasil em relação aos cinco países líderes (1982-2016)

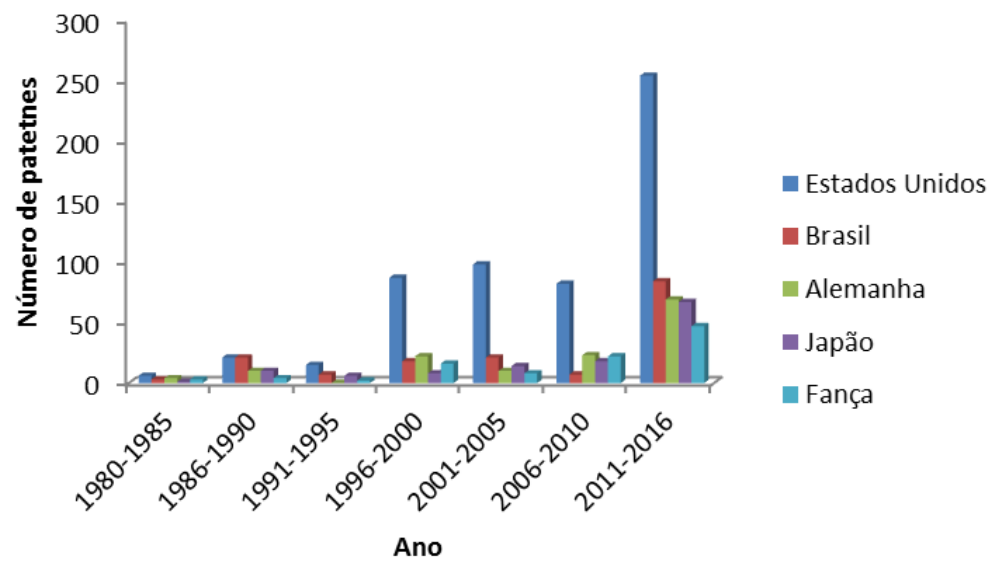

Fonte: Autores

Verifica-se que os cinco principais países de origem apresentaram tendências próximas de patenteamento em biotecnologia até o início da década de 1990. Depois desse período, os Estados Unidos mostraram um desenvolvimento superior aos demais países, com um notório crescimento a partir de 1996. Este salto significativo está associado, principalmente, ao número elevado de patentes na área farmacêutica $(35,5 \%)$ e ao fato de o país liderar o ranking de pedidos pipeline concedidos, com 57,7\% dos registros.

No período de 2011 a 2016 os EUA avançaram em um ritmo frenético, mas se verificou uma taxa de crescimento para todos os cinco países. A Alemanha e o Japão estiveram bem próximos, enquanto a França ocupou a última posição. O Brasil chegou ao segundo lugar do ranking, ainda que mantivesse uma distância significativa em relação aos EUA.

A Figura 6 apresenta a produtividade dos Estados brasileiros no patenteamento em biotecnologia (1982-2016). 
Figura 6 - Distribuição das patentes concedidas em biotecnologia pelas unidades federativas no Brasil (1982-2016).

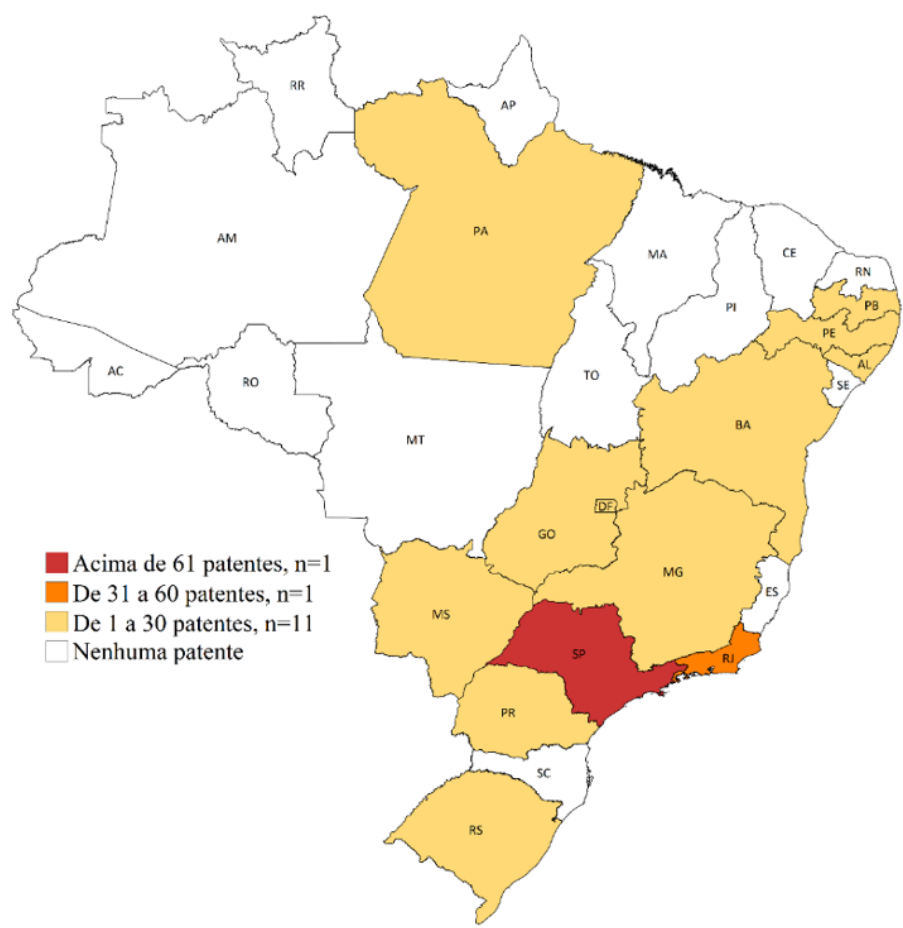

Nota: $\mathrm{SP}=$ São Paulo, RJ = Rio de Janeiro, $\mathrm{MG}=$ Minas Gerais, $\mathrm{RS}=$ Rio Grande do Sul, $\mathrm{PR}=$ Paraná, DF $=$ Distrito Federal, $\mathrm{AL}=$ Alagoas, $\mathrm{BA}=$ Bahia, $\mathrm{PB}=$ Paraíba, $\mathrm{PE}=$ Pernambuco, GO = Goiás, MS = Mato Grosso do Sul, PA = Pará, ES = Espírito Santo, SC $=$ Santa Catarina, $\mathrm{CE}=$ Ceará, $\mathrm{RN}=$ Rio Grande do Norte, $\mathrm{SE}=$ Sergipe, $\mathrm{PI}=$ Piauí, $\mathrm{MA}=$ Maranhão, $\mathrm{TO}=$ Tocantins, $\mathrm{MT}=\mathrm{Mato}$ Grosso, $\mathrm{RO}=$ Rondônia, $\mathrm{AC}=\mathrm{Acre}, \mathrm{AP}=$ Amapá, $\mathrm{AM}=$ Amazonas, $\mathrm{RR}=$ Roraima .

Fonte: Autores

A análise geográfica sobre as unidades federativas revela que só o Estado de São Paulo acumula 58,4\% da produção tecnológica na área, seguida pelo Rio de Janeiro (20,5\%) e Minas Gerais (8,7\%). Pode-se observar, também, uma forte concentração desta tecnologia $(87,6 \%)$ na região sudeste do país, com destaque para as universidades, que detiveram 40,4\% dos registros de patentes.

Por outro lado, observou-se que mais da metade dos Estados brasileiros não possuíam patentes concedidas em biotecnologia, principalmente em regiões com elevada biodiversidade, como é caso da região norte do país, composta pelos Estados do Acre, Amapá, Amazonas, Tocantins, Rondônia e Roraima, todos sem proteção de patentes em biotecnologia.

A análise sob a dimensão regional revelou que a maioria das regiões brasileiras (Nordeste, Centro-Oeste, Sul, Sudeste) apresentou uma forte vocação para a área de biotecnologia industrial, especificamente para a produção de biocombustível. Neste contexto, destacam-se importantes políticas governamentais que destinaram uma grande quantidade de 
recursos públicos. Estas políticas energéticas tornaram os biocombustíveis comercialmente relevantes para a indústria e promoveram uma verdadeira corrida tecnológica no setor (Florencio, Abud, \& Oliveira Jr, 2019).

A Figura 7 mostra que a maioria das patentes em biotecnologia no Brasil é protegida por empresas privadas (81\%), seguida por universidades (6\%), instituições públicas (4\%) e pessoa física (3\%).

Figura 7 - Perfil dos titulares de patentes em biotecnologia no Brasil, com destaque para os titulares parceiros.

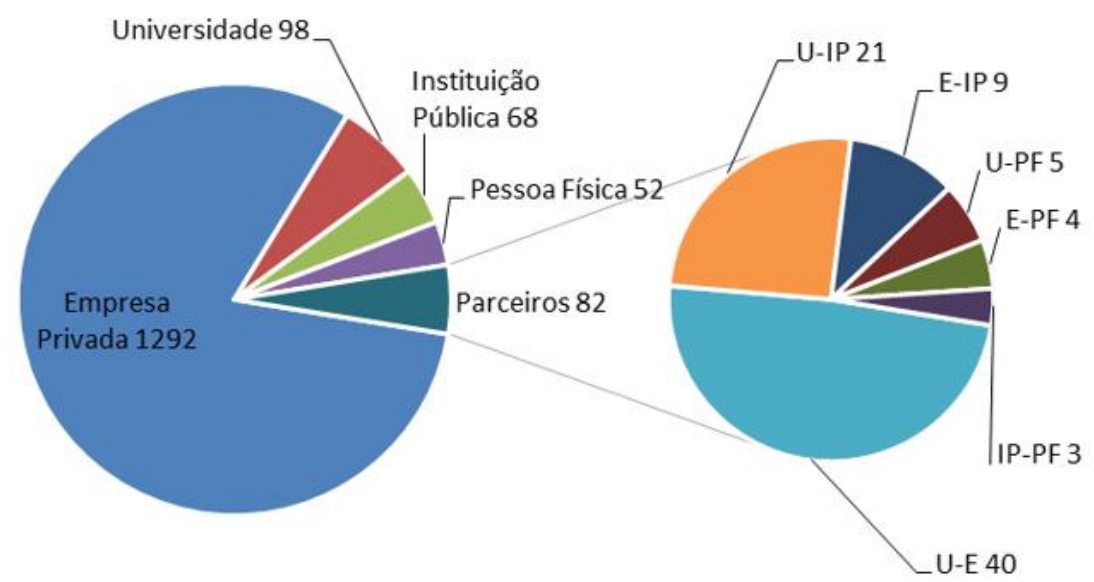

Nota: U-IP = Universidade e Instituição Pública, E-IP = Empresa e Instituição Pública, U-PF = Universidade e Pessoa Física, E-PF = Empresa e Pessoa Física, IP-PF = Instituição Pública e Pessoa Física, U-E = Universidade e Empresa.

Fonte: Autores

Cabe enfatizar que a grande maioria das empresas privadas com patentes em biotecnologia no Brasil (94\%) é estrangeira, notadamente empresas americanas.

$\mathrm{O}$ alto número de titulares de patentes observado em empresas privadas pode indicar um cenário otimista para o sistema nacional de inovação em biotecnologia, que passa a contar com maiores chances de aplicação comercial para os novos produtos e processos gerados. Por outro lado, o grande número de titulares estrangeiros coloca o Brasil na posição de consumidor de biotecnologia.

Ao se analisar o número de depositantes por patente, percebeu-se que $91 \%$ dos documentos apresentaram um único titular, enquanto as demais patentes possuíram uma média de dois depositantes. Isto mostrou que o setor de biotecnologia apresenta um baixo índice de colaboração dos titulares de patentes, com apenas $9 \%$ em relação ao total de documentos recuperados. O maior número de parceiros titulares foi observado entre 
universidade e empresa privada $(2,5 \%)$, seguido de universidade e instituição pública $(1,3 \%)$ e empresa privada e instituição pública $(0,6 \%)$.

Um total de 784 titulares detiveram a proteção de 1.592 patentes em biotecnologia no Brasil, no período de 1982 a 2016, sendo a maior parte deles (71,8\%) com registros de apenas uma patente, enquanto 28,2\% possuíram pelo menos duas patentes. Essa dispersão da produção tecnológica em biotecnologia segue a Lei de Lotka (1926), onde muitos autores publicaram próximo de um artigo, e um reduzido número de pesquisadores, supostamente de maior prestígio em uma área do conhecimento, publicaram muitos (Dabi et al., 2016).

A Tabela 2 apresenta os 20 principais titulares de patentes em biotecnologia identificados no estudo. A maioria é empresa privada estrangeira, localizadas principalmente na América do Norte e na Europa.

Tabela 2 - Principais titulares de patentes em biotecnologia no Brasil entre 1982 a 2016.

\begin{tabular}{lcc}
\hline \multicolumn{1}{c}{ Nome do titular } & País & \% Patentes \\
\hline Ajinomoto Co., Inc. & Japão & 4,08 \\
Novozymes S/A & Dinamarca & 3,14 \\
Monsanto Technology & Estados Unidos & 2,64 \\
Bayer & Bélgica/ Alemanha & 2,14 \\
Sanofi-Aventis & Alemanha & 1,95 \\
F. Hoffmann-La Roche & Suíça & 1,88 \\
Wyeth Holdings Corporation & Estados Unidos & 1,32 \\
Genentech & Estados Unidos & 1,26 \\
Syngenta & Suíça & 1,19 \\
Universidade de São Paulo (USP) & Brasil & 1,13 \\
Merial & França & 1,07 \\
E.I. du Pont de Nemours and Company & Estados Unidos & 0,94 \\
Eli Lilly and Company & Estados Unidos & 0,94 \\
Basf Aktiengesellschaft & Alemanha & 0,94 \\
Intervet International B.V. & Holanda & 0,88 \\
Universidade Estadual de Campinas (UNICAMP) & Brasil & 0,88 \\
Société Des Produits Nestlé S.A & Suíça & 0,88 \\
Evonik Degussa GmbH & Alemanha & 0,82 \\
Dow Agrosciences & Estados Unidos & 0,82 \\
Smithkline Beecham Biologicals S.A. & Bélgica & 0,82 \\
Outros titulares & & 69,16 \\
\hline Total & & 100,00 \\
\hline
\end{tabular}

Fonte: Autores

Destaca-se que as principais instituições concentraram 30,8\% do total da produção biotecnológica no Brasil.

A japonesa Ajinomoto foi uma empresa que mais apresentou biotecnologias patenteadas, com cerca de $4 \%$ da produção total na área. Salienta-se que a maior parte das patentes $(75 \%)$ da Ajinomoto Co, Inc. está relacionada ao setor de alimentos, em especial à produção de aditivos alimentares. 
A Novozymes S/A, multinacional dinamarquesa, foi a segunda do ranking, com 3,1\% das patentes concedidas em biotecnologia, sendo $96 \%$ destas voltadas à produção de enzimas ou de microrganismos com diferentes finalidades. A Monsanto Technology foi a terceira maior instituição, com 2,64\% das biotecnologias protegidas, tratando, na sua maioria, de processos de obtenção de plantas geneticamente modificadas.

A biotecnologia é uma área multidisciplinar com aplicação em diferentes setores da economia. Nos documentos analisados, classificou-se a produção tecnológica em biotecnologia no Brasil nos setores de biotecnologia em saúde, industrial, agrícola e ambiental. A Figura 8 mostra que este desenvolvimento está concentrado na área de saúde (46,3\%), em detrimento do setor ambiental, que apresenta apenas 2,3\% do total das patentes concedidas em biotecnologia.

Figura 8 - Distribuição das patentes por setores de aplicação da biotecnologia

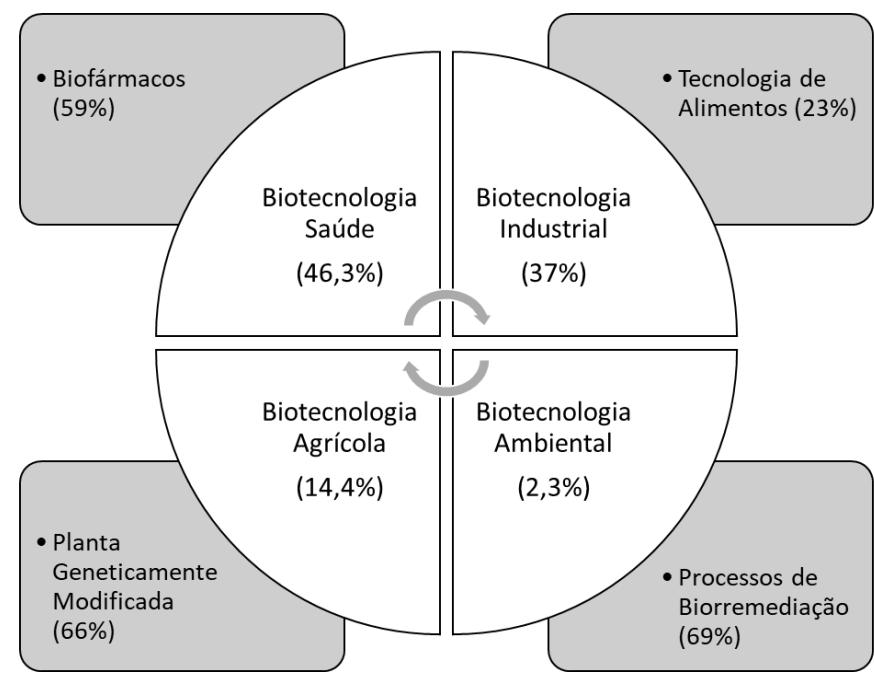

Fonte: Autores.

A fim de obter uma maior compreensão da dinâmica de desenvolvimento da biotecnologia no Brasil, fez-se uso da análise de redes sociais, onde o recorte temporal dos dados foi pautado nos últimos dez anos (2007 a 2016), após aprovação da PDB no país.

\subsection{Rede de Colaboração da Biotecnologia em Saúde}

A análise da colaboração tecnológica em biotecnologia saúde no Brasil foi realizada, inicialmente, com os titulares de duas ou mais patentes. A rede institucional em biotecnologia 
saúde no Brasil compreendeu 77 nós, distribuídos em três clusters principais (Figura 9), chamando a atenção para o elevado número de nós isolados, demonstrando que o patenteamento em parceria é pouco visado.

Figura 9 - Rede de Produção Tecnológica em Biotecnologia Saúde no Brasil entre as instituições com duas ou mais patentes (2007-2016).
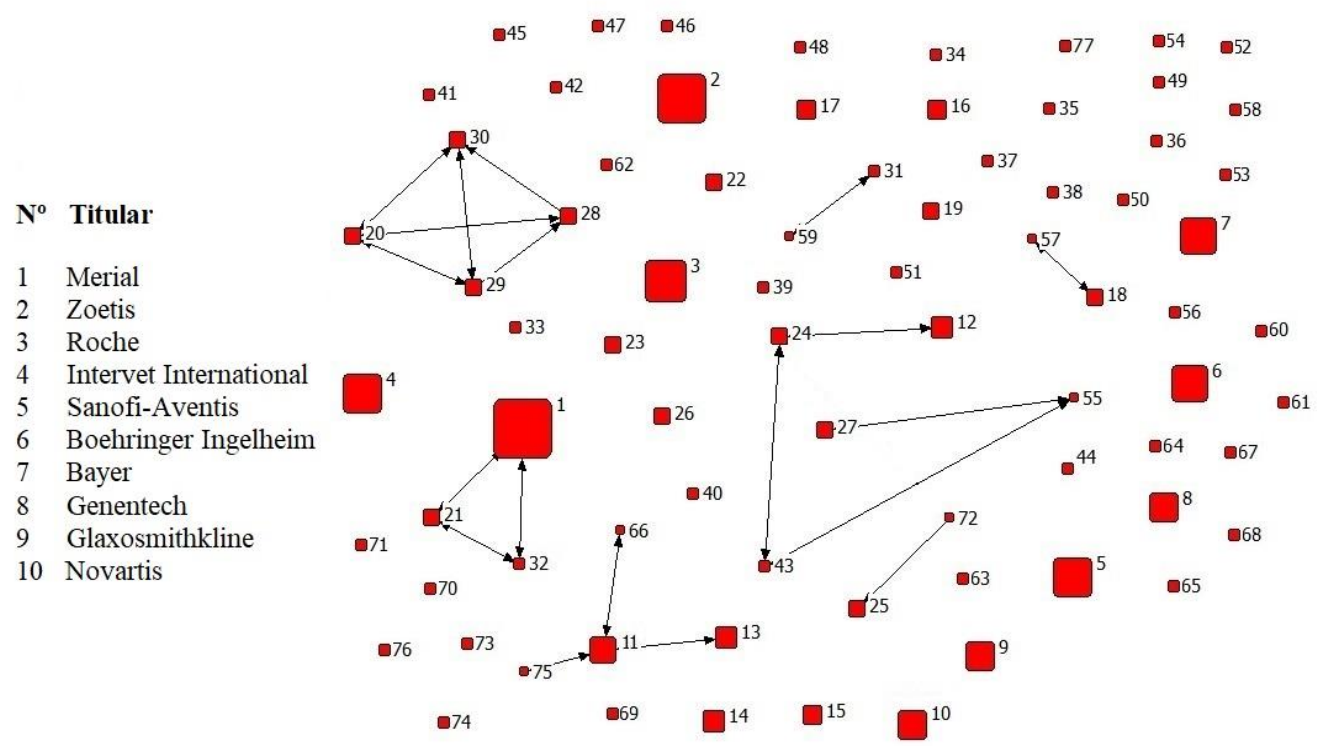

Fonte: Autores

Além disso, os dez principais titulares de patentes em biotecnologia saúde estabeleceram pouco ou nenhum vínculo com os demais atores da rede, sendo cerca de apenas $2 \%$ das patentes registradas em conjunto.

O maior cluster tratou das parcerias entre os titulares não residentes. Aventis Pharma (12), Centro Nacional da Pesquisa Científica da França - CNRS (24), Instituto Pasteur (43), Instituto Nacional de Pesquisa Médica e de Saúde da França - INSERM (55) e Merck Serono (27) possuíram 4 ligações. As parcerias tomadas em conjunto corresponderam à área farmacêutica com foco na saúde humana, com patentes de medicamentos, vacina e kits diagnósticos para o tratamento de infecções e outras aplicações.

O segundo maior cluster apresentou uma forte interação. As instituições University of Massachusetts Medical Center (20), Instituto Max Planck (28), Massachusetts Institute of Technology (29) e a Whitehead Institute for Biomedical Research (30), com 5 ligações, registraram todas as suas invenções em conjunto. Já o terceiro maior cluster correspondeu a titulares residentes no Brasil. A Fundação de Amparo à Pesquisa do Estado de São Paulo - 
FAPESP (11), a USP (13), a Biolab Farmacêutica (66) e a Universidade Federal de São Paulo - UNIFESP (75), com 3 ligações, registraram patentes com diferentes finalidades. Destaca-se que a FAPESP foi identificada como ponte de ligação entre os atores do grupo.

Ao analisar os indicadores de redes (Tabela 3), verificou-se que o valor da densidade para dados ditocomizados foi: em torno de $0,5 \%$, com desvio de $7,4 \%$, o que revela uma fraca interação de comunicação entre as instituições.

Tabela 3 - Medidas de centralidade da rede de produção tecnológica em biotecnologia saúde no Brasil (2007-2016).

\begin{tabular}{ccccc}
\hline Métricas/Rede & Média & $\begin{array}{c}\text { Desvio } \\
\text { padrão }\end{array}$ & $\begin{array}{c}\text { Coeficiente } \\
\text { Variação }\end{array}$ & Instituição de destaque \\
\hline Grau de Centralidade & 0,416 & 0,843 & $202,6 \%$ & UMMC, Whitehead Institute, Max- \\
& & & & Planck, MIT \\
Grau de Intermediação & 0,130 & 0,652 & $501,5 \%$ & Instituto Pasteur \\
Grau de Proximidade & 1,341 & 0,021 & $1,6 \%$ & Instituto Pasteur \\
Densidade & $0,5 \%$ & $7,4 \%$ & - & - \\
Índice de Centralização & $3,49 \%$ & - & - & - \\
\hline
\end{tabular}

Fonte: Autores.

A medida de centralização apresentou um índice de 3,49\%, sugerindo que a rede não apresenta instituições com alto poder de interação.

O grau de centralidade mostrou uma média de 0,416 e um desvio de 0,843, indicando que a interação de cada instituição não chega a uma ligação com outros. Isto mostra que na rede existe uma baixa probabilidade de captação de uma informação por todos os atores.

Ainda que o Instituto Pasteur tenha se destacado em relação aos demais atores como um canal de informação, o grau de proximidade e intermediação apresentaram valores quase inexpressíveis, 0,130 e 1,341 respectivamente, sugerindo uma falta de atores-chave que exercessem um papel claro de intermediação do conhecimento na rede.

\subsection{Rede de Colaboração da Biotecnologia Industrial}

A partir da exploração das parcerias entre os titulares com duas ou mais patentes, a rede de colaboração tecnológica em biotecnologia industrial no Brasil é apresentada na Figura 10. 
A rede de colaboração em biotecnologia industrial possuiu 48 instituições e 2 clusters principais, havendo uma predominância de nós isolados (73\%), fato que remete à pouca expressividade de colaboração entre os atores da rede.

O cluster principal foi formado por titulares residentes no Brasil. A Universidade Federal de São Carlos - UFSCAR (15), a FAPESP (18), o Instituto de Pesquisas Tecnológicas de São Paulo - IPT (38) e a empresa Phb Industrial S/A (40) formaram parcerias entre si para a produção de biorreatores ou biopolímeros. O segundo maior cluster compreendeu as multinacionais DuPont (5), Genencor International (10) e Tate \& Lyle Ingredients (21). Destaca-se que a DuPont e a Genencor International possuem 4 patentes em conjunto voltadas para a produção de compostos orgânicos com aplicação na indústria de alimentos e outros setores.

Figura 10 - Rede de produção tecnológica em biotecnologia industrial no Brasil entre as instituições com duas ou mais patentes (2007-2016).

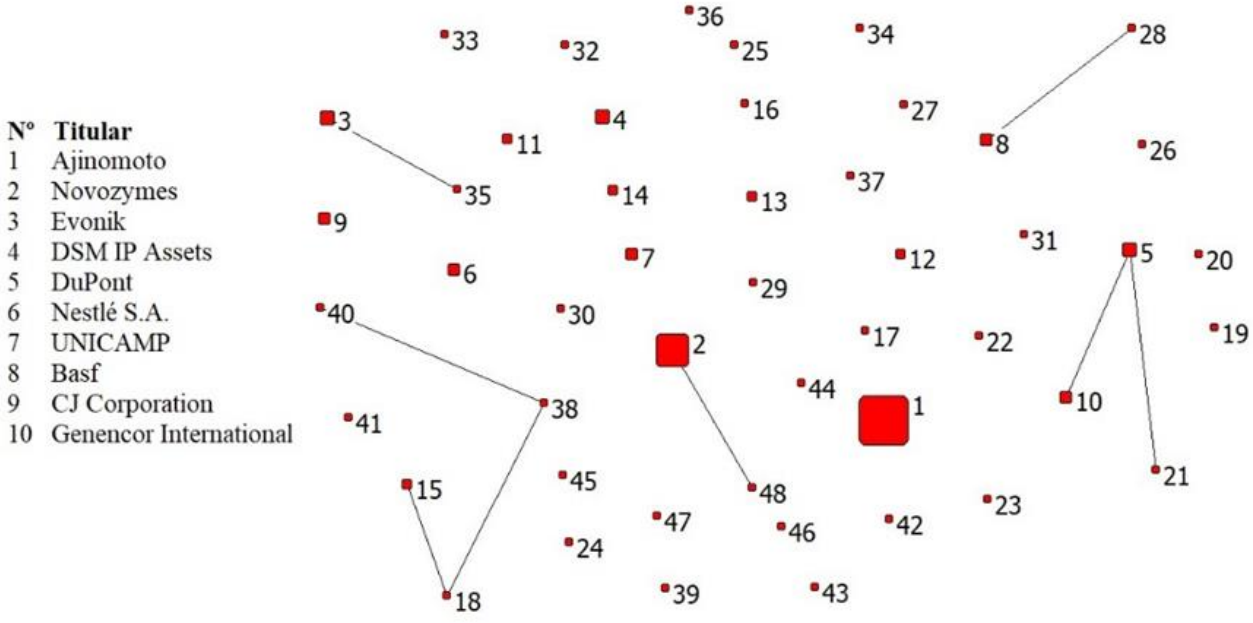

Fonte: Autores.

Quanto aos principais titulares (top 10), além da DuPont foi possível perceber que a Novozymes (2), a Evonik (3) e a Basf (8) estabeleceram parcerias com outros na rede. Identificou-se ainda a existência de uma tímida colaboração internacional $(0,6 \%)$ entre as empresas da Alemanha e instituições da Dinamarca e Austrália. Gigante, Rigolin \& Marcelo (2012) apontaram que a fraca colaboração internacional é mais visível nas áreas de PD\&I tecnológica, as quais requerem experimentações, laboratórios e uso de equipamentos dedicados e se defrontam com dificuldades relacionadas, principalmente, às distâncias 
geográficas, aos custos de deslocamento e às barreiras linguísticas. A Tabela 4 mostra as medidas de centralidade da rede de colaboração em biotecnologia industrial no Brasil.

Tabela 4 - Medidas de centralidade da rede de colaboração tecnológica em biotecnologia industrial no Brasil (2007-2016).

\begin{tabular}{lcccc}
\hline Métricas/Rede & Média & $\begin{array}{c}\text { Desvio } \\
\text { padrão }\end{array}$ & $\begin{array}{c}\text { Coeficiente } \\
\text { Variação }\end{array}$ & Instituição de destaque \\
\hline Grau de Centralidade & 0,333 & 0,709 & $212,91 \%$ & IPT, FAPESP e DuPont \\
Grau de Intermediação & 0,104 & 0,420 & $403,85 \%$ & IPT e FAPESP \\
Grau de Proximidade & 2,167 & 0,040 & $1,85 \%$ & IPT e FAPESP \\
Densidade & $0,7 \%$ & $8,4 \%$ & - & - \\
Índice de Centralização & $3,70 \%$ & - & - & - \\
\hline
\end{tabular}

Fonte: Autores.

O índice de centralização $(3,7 \%)$ foi significativamente baixo e, somado a isso, o grau de centralidade apresentou uma média de 0,333 e desvio de 0,709. Estes dados evidenciam uma alta dispersão entre as instituições, não sendo possível apontar atores que exerceram um papel central na rede.

Os valores do grau de proximidade $(2,167)$ e de intermediação $(0,104)$ indicaram a falta de instituições com posição de controle do fluxo de informação entre diferentes atores. Por meio da densidade, verifica-se que a capacidade de conexão entre todos os atores é de $0,7 \%$, com desvio $8,4 \%$.

\subsection{Rede de Colaboração da Biotecnologia Agrícola}

A colaboração em biotecnologia agrícola no Brasil (Figura 11) possui uma rede composta por 61 nós (instituições titulares de patentes), sendo apenas 7\% destes apresentando patentes em conjunto. Isto mostra que, embora a colaboração seja importante para difundir a inovação e aumentar a produção científica ou tecnológica, é um elemento ainda pouco visado entre os titulares de patentes em biotecnologia agrícola no Brasil. 
Figura 11 - Rede institucional de produção tecnológica em biotecnologia agrícola no Brasil (2007-2016).
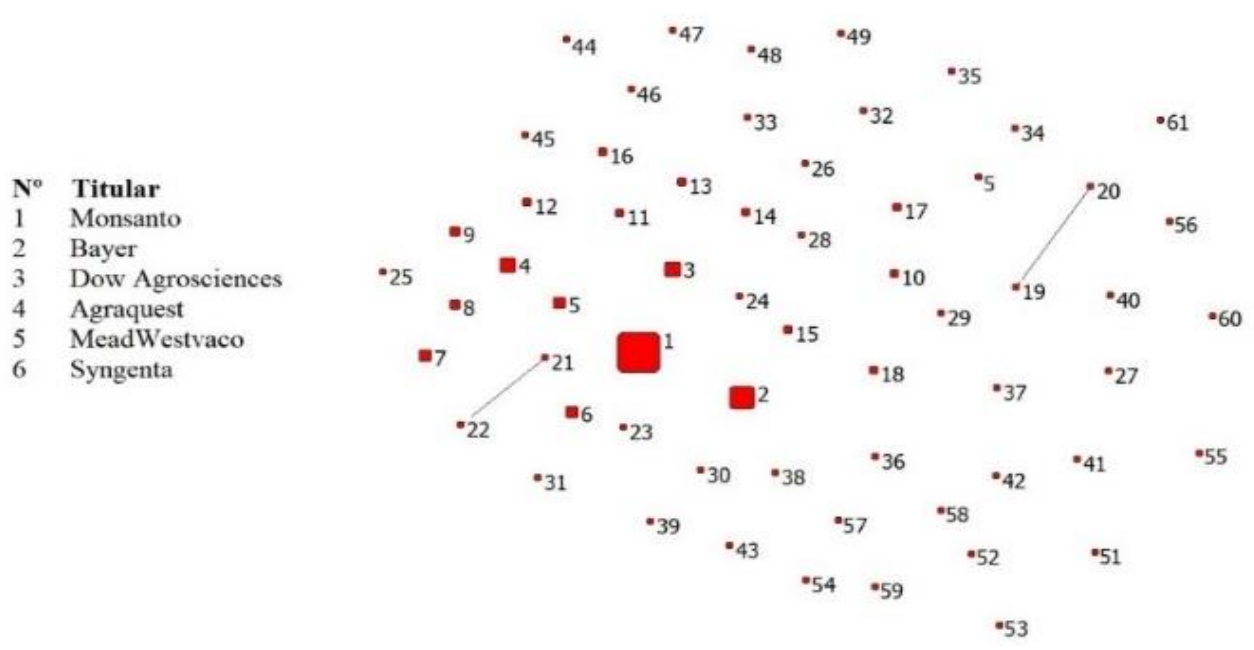

Fonte: Autores.

Os dados mostraram que os principais detentores de patente (top 6) não possuiam vínculo com outros e as poucas parcerias existentes ocorreram entre instituição pública, governo e/ou universidade de diferentes nacionalidades (Brasil, Estados Unidos, Canadá e Holanda). A rede de colaboração apresentou densidade de 1\%, indicando uma fraca conexão entre todos os atores.

\subsection{Rede de Colaboração da Biotecnologia Ambiental}

A Figura 12 apresenta a colaboração tecnológica em biotecnologia ambiental no Brasil por ARS, elaborada a partir de uma matriz e visualizada por meio do software NetDraw. 
Figura 12 - Rede institucional de produção tecnológica em biotecnologia ambiental no Brasil (2007-2016).

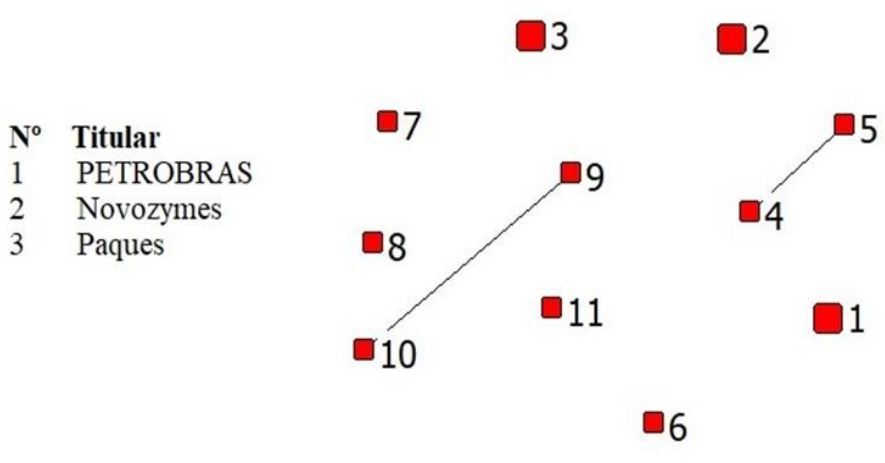

Fonte: Autores.

A rede foi composta por apenas 11 instituições, com predominância de nós isolados (64\%), evidenciando que o patenteamento em parceria é um elemento pouco explorado na área. As colaborações existentes foram formadas entre universidades brasileiras e agências de fomento e/ou institutos públicos, em parcerias voltadas para o desenvolvimento de tecnologias de biorremediação. Constatou-se, ainda, que os três maiores detentores de patentes na área (PETROBRAS, Novozymes e Paques) não possuem vínculos uns com os outros.

Quanto aos indicadores de centralidade da rede, identificou-se uma densidade de $3,6 \%$, com desvio padrão de $18,7 \%$. O grau de centralidade, por sua vez, apresentou uma média de 0,364 e desvio de 0,481, indicando que a média das interações entre os atores não chega a uma ligação com outros.

\section{Considerações Finais}

Ações e políticas de C, T\&I para o desenvolvimento tecnológico da biotecnologia no Brasil foram traçadas há mais de uma década mas, apesar disto, não é possível observar um valor significativo de biotecnologias patenteadas (1.592 concessões). Este número se torna ainda mais ínfimo quando se observam os resultados dos titulares residentes (161 concessões), cujo baixo desempenho pode ser explicado, pelo menos em parte, pelo enorme atraso no exame dos pedidos de patentes, que obtiveram uma média geral de 10,6 anos. De maneira geral, os resultados mostraram uma média geral de atraso de concessão mais baixa para residentes do que não residentes, sugerindo que as instituições estrangeiras lidam com 
maiores dificuldades em relação aos depositantes nacionais por, muitas vezes, possuírem uma cultura de proteção da biotecnologia diferente do que é aceitável pela legislação brasileira.

Os dados também revelaram que o sistema de patente brasileiro tem beneficiado enormemente os depositantes estrangeiros, principalmente por meio dos registros pipeline, que representaram um total de $13 \%$ das patentes concedidas, tornando o mercado de proteção da biotecnologia no Brasil amplamente dominado por titulares não residentes, em todas as áreas setoriais. A maioria dos principais titulares são empresas multinacionais situadas em países desenvolvidos, com elevados investimentos em P,D\&I. Por outro lado, as patentes de residentes pertencem, em sua grande maioria, às instituições públicas, sugerindo a necessidade de ações governamentais para disseminar e fortalecer a cultura da propriedade intelectual no país, possibilitando treinamentos em relação à proteção das biotecnologias tanto para as instituições públicas quanto para o setor empresarial.

Outro ponto que merece atenção é o alto percentual de patentes extintas em função da falta de pagamento da retribuição anual, em cerca de $10 \%$ das concessões. Essas tecnologias possivelmente não possuem aplicações comerciais relevantes, indicando que os dados de patentes são um indicador imperfeito da inovação em biotecnologia.

A ARS nos distintos setores revelou uma fraca colaboração entre os diferentes tipos de titulares de patentes (empresa, academia, instituição pública). Os principais detentores de biotecnologias nas diversas áreas setoriais estabeleceram pouco ou nenhum vínculo com as instituições brasileiras, com o patenteamento em parceria sendo um elemento ainda pouco explorado. Diante desse cenário, chama-se a atenção para importantes políticas de fortalecimento da relação universidade-empresa, como as Leis de Inovação nº 10.973/2004 e 13.243/2016, o Fundo Setorial Verde Amarelo (FVA) e o Programa de Formação de Recursos Humanos em Áreas Estratégicas (RHAE). Espera-se que as ações desenvolvidas por esses programas, ao longo prazo, produzam efeitos positivos para melhorar o ambiente de colaboração e desenvolvimento tecnológico envolvendo a biotecnologia no Brasil.

Por fim, trabalhos futuros poderiam conduzir uma análise da produção tecnológica da biotecnologia por meio de dados de pedidos de depósitos de patentes a fim de identificar tecnologias promissoras e emergentes. Além disso, a adoção de outros indicadores bibliométricos, como medidas de proteção e citação, é útil em novas pesquisas que objetivam estudar a inovação em biotecnologia. 


\section{Agradecimentos}

O presente trabalho foi realizado com apoio da Coordenação de Aperfeiçoamento de Pessoal de Nível Superior - Brasil (CAPES) - Código de Financiamento 001.

\section{Referências}

Banerjee, P, Gupta, BM \& Garg, KC. (2000). Patent statistics as indicators of competition an analysis of patenting in biotechnology. Scientometrics, 47(1), 95-116, doi: 10.1023/A:1005669810018.

Belda, I, Penas, G, Alonso, A, Marquina, D, Navascués, A \& Santos, A. (2014). Biotech patents and science policy: the Spanish experience. Nature Biotechnology, 32(1), 59-61, doi: 10.1038/nbt.2781.

Brasil. (1996). Lei n. 9.279, de 14 de maio de 1996. Regula direitos e obrigações relativos à propriedade industrial. Diário Oficial [da] República Federativa do Brasil 14 mai 1996.

Brasil. A revisão da lei de patentes: inovação em prol da competitividade nacional. Brasília: Edições Câmara, 2013. 405p. [acesso em 10 mai 2020]. Disponível em: http://bd.camara.gov.br/bd/handle/bdcamara/14796.

Buttow, ME \& Steindel, M. (2012). Patent application in biotechnology at subclass C12N in Brazil at the period of 2001 to 2005. Brazilian Archives of Biology and Technology, 55(3), 341-348, doi:10.1590/S1516-89132012000300003.

Costa, SCC, Neto, AG, \& De Gutiérrez, IEM. (2012). Ensino, empresas e patentes em biotecnologia no país. GEINTEC-Gestão, Inovação e Tecnologias, 2(2), 138-153, doi:10.7198/geintec.v2i2.32.

Costa, BMG, Florencio, MNS, \& Oliveira Jr, AM. (2018). Analysis of technological production in biotechnology in northeast Brazil. World Patent Information, 52, 42-49, doi: 10.1016/j.wpi.2018.01.006. 
Dabi, Y, Darrigues, L, Katsahian, S, Azoulay, D, Antonio, M, \& Lazzati, A. (2016). Publication trends in bariatric surgery: a bibliometric study. Obesity Surgery, 26(11), 26912699, doi:10.1007/s11695-016-2160-x.

Dias, F, Delfim, F, Drummond, I, Carmo, AO, Barroca, TM, Horta, CC, \& Kalapothakis, E. (2012). Evaluation of Brazilian biotechnology patent activity from 1975 to 2010. Recent Patents on DNA \& Dene Sequences, 6(2), 145-159, doi:10.2174/187221512801327424.

Florencio, MNS, Abud, AKS, \& Oliveira Jr, AM. (2019). Análise da produção tecnológica em biotecnologia industrial no Brasil. Revista Tecnologia e Sociedade, 15(37), 403-416, doi:10.3895/rts.v15n37.9697.

Garcez Jr, SS, \& Moreira, JJS. (2017). O backlog de patentes no Brasil: o direito à razoável duração do procedimento administrativo. Revista Direito GV, 13(1), 171-203.

Gigante, LC, Rigolin, CCD, \& Marcelo, JF. (2012). Redes sociais de produção e colaboração tecnológica para o descarte de resíduos de equipamentos elétricos e eletrônicos. AtoZ: Novas práticas em informação e conhecimento, 1(2), 52-64.

Instituto Nacional da Propriedade Industrial. Anuário estatístico da propriedade industrial 2000-2016. INPI: 2017. [acesso em 10 mai 2020]. Disponível em: http://www.inpi.gov.br/sobre/estatisticas.

Instituto Nacional da Propriedade Industrial. Exame prioritário "Patentes Verdes" se torna serviço permanente do INPI. INPI: 2016. [acesso em 10 mai 2020]. Disponível em: http://www.inpi.gov.br/noticias/Patentes\%20Verdes.

Instituto Nacional da Propriedade Industrial. Diretriz de Exame de Pedidos de Patente na Área de Biotecnologia. 2015. [acesso em 10 mai 2020]. Disponível em: http://www.inpi.gov.br/sobre/arquivos/resolucao_144-2015_-_diretrizes_biotecnologia.pdf.

Jannuzzi, AHL, \& Vasconcellos, AG. (2017). Quanto custa o atraso na concessão de patentes de medicamentos para a saúde no Brasil? Cadernos Saúde Pública, 33(8), p. e00206516, doi:10.1590/0102-311x00206516. 
Matias-Pereira, J. (2011). A gestão do sistema de proteção à propriedade intelectual no Brasil é consistente? Revista de Administração Pública, 45(3), 567-590, doi:10.1590/S003476122011000300002 .

Mendes, L, Amorim-Borher, B, \& Lage, C. (2013). Patent Applications on Representative Sectors of Biotechnology in Brazil: an Analysis of the Last Decade. Journal of Technology Management \& Innovation, 8(4), 91-102, doi:10.4067/S0718-27242013000500009.

Mejer, M, \& Van Pottelsberghe De La Potterie, B. (2011). Patent backlogs at USPTO and EPO: systemic failure vs. deliberate delays. World Patent Information, 33, 122-127, doi:10.1016/j.wpi.2010.12.004.

Miranda, P.H.M.V., Silva, F.V.N., \& Pereira, A.M.C. Perguntas e respostas sobre patentes pipeline: como afetam sua saúde? Rio de Janeiro: ABIA, 2009. 21p.[acesso em 10 mai 2020]. Disponível em: http://www.abiaids.org.br/_img/media/PergResp_PIPELINE_PT.pdf.

Nelson, AJ. (2009). Measuring knowledge spillovers: what patents, licenses and publications reveal about innovation diffusion. Research Policy, 38(6), 994-1005, doi:10.1016/j.respol.2009.01.023.

Okano, MT. (2020). Análise bibliométrica das empresas sociais: qual o seu impacto na produção acadêmica? Research, Society and Development, 9(3), e73932435, doi:10.33448/rsd-v9i3.2435

Pereira, AS, Shitsuka, DM, Parreira, FJ \& Shitsuka, R. (2018). Metodologia da pesquisa científica. [e-book]. Santa Maria. Ed. UAB/NTE/UFSM. Acesso em: 9 maio 2020. Disponível em: https://repositorio.ufsm.br/bitstream/handle/1/15824/Lic_Computacao_MetodologiaPesquisa-Cientifica.pdf?sequence $=1$.

Pinto, PE, Vallone, A, Honores, G, \& González, H. (2017). The dynamics of patentability and collaborativeness in Chile: An analysis of patenting activity between 1989 and 2013. World Patent Information, 49, 52-65, doi:10.1016/j.wpi.2017.05.004. 
Popp, D, Juhl, T, \& Johnson, DKN. (2004). Time in Purgatory: Determinants of the Grant Lag for U.S. Patent Applications. Topics in Economic Analysis \& Policy, 4(1), 1-48, doi: $10.3386 /$ w9518.

Tijssen, RJW. (2011). Global and domestic utilization of industrial relevant science: patent citation analysis of science technology interactions and knowledge flows. Research Policy, 30(1), 35-54, doi:10.1016/S0048-7333(99)00080-3.

Van Beuzekom, B., \& Arundel, A. OECD Biotechnology Statistics. OECD, 2009. [acesso em 10 mai 2020]. Disponível em: https://www.oecd.org/sti/42833898.pdf.

\section{Porcentagem de contribuição de cada autor no manuscrito}

Márcio Nannini da Silva Florêncio - 32,5\%

Ana Karla de Souza Abud - 22,5\%

Benedita Marta Gomes Costa - 22,5\%

Antonio Martins de Oliveira Junior - 22,5\% 